\title{
Supralevator Total Pelvic Exenteration Without Colostomy - Case Report
}

\author{
Marian Botoncea1, Claudiu Molnar Varlam², Adrian Chiujdea³, Călin Molnar' \\ 1 Clinic of Surgery I, County Emergency Clinical Hospital, Tîrgu Mureș, Romania \\ 2 Clinic of Urology, County Clinical Hospital, Tîrgu Mureș, Romania \\ ${ }^{3}$ Clinic of Obstetrics and Gynecology I, County Emergency Clinical Hospital, Tîrgu Mureș, Romania
}

\section{CORRESPONDENCE \\ Claudiu Molnar Varlam \\ Str. Gheorghe Marinescu nr. 38 \\ 540139 Tîrgu Mureș, Romania \\ Tel: +40 744152022 \\ E-mail:molgyn@yahoo.com}

\section{ARTICLE HISTORY}

Received: June 15, 2017

Accepted: July 23, 2017
Marian Botoncea • Str. Gheorghe Marinescu nr. 38 540139 Tîrgu Mureș, Romania. Tel: +40 758468909 . E-mail: botonceam@gmail.com

Călin Molnar • Str. Gheorghe Marinescu nr. 38 540139 Tîrgu Mureș, Romania. Tel: +40 722696610. E-mail:molnar.calin@yahoo.com

Adrian Chiujdea - Str. Gheorghe Marinescu nr. 50, 540136 Tîrgu Mureș, Romania. Tel: +40 743169579. E-mail: achiujdea@yahoo.com

\begin{abstract}
Background: Pelvic exenteration is an ultra-radical surgical procedure described by Brunschwig in 1948, which attempts to surgically cure patients with recurrent pelvic cancer after radiotherapy. Several variants of pelvic exenteration are described that allow a more limited or extensive resection, depending on the stage of the disease. Case report: We report the case of a 54-year-old woman, who was diagnosed with a tumoral rectovaginal fistula after a recurrent cervical cancer that had been treated with a total hysterectomy with bilateral adnexectomy and a left percutaneous nephrostomy, as well as interaortocaval lymph node resection. The patient had undergone a supralevator total pelvic exenteration with pelvic and interaortocaval lymphadenectomy. The reconstruction process included right ureterostomy, left nephrostomy, and colocutaneous anal anastomosis (Parks procedure). Conclusions: Supralevator total pelvic exenteration provides hope for cure in patients with pelvic malignancies that reappear after radiotherapy. The restoration of the digestive tract and avoiding colostomy with a colocutaneous anastomosis increases the quality of life in these cases.
\end{abstract}

Keywords: supralevator pelvic exenteration, colocutaneous anastomosis, cervical cancer

\section{INTRODUCTION}

Pelvic exenteration was first introduced by Alexander Brunschwig in 1948 as "the most radical surgical attack so far described for pelvic cancer". ${ }^{1}$ His surgical method comprises a one-staged abdominal-perineal operation with a total en masse resection of the pelvic organs, placement of an end-colostomy, and bilateral ureteral implantation above the colostomy. Brunschwig introduced the method as a way for symptomatic palliative care in subjects with advanced gynecological malignancies that do not respond to radiation therapy, who experience subsequent complications including fistula, infection, or pain. He performed these operations with a high perioperative mortality rate of $23 \% .^{1}$

Considerable progress has been made in the management of these patients as the method underwent substantial advances, and it was reported that the physical and psychological morbidity of exenterative surgery has decreased from $23 \%$ 
to $3-5 \% .^{2}$ Heriot et al. reported morbidity and mortality rates of $27 \%$ and $0.6 \%$ respectively. ${ }^{3}$ These improvements can also be credited to developments in intraoperative and perioperative treatments, including a better patient selection, the routine use of prophylactic antibiotherapies, venous thromboembolism prevention, anesthesia monitoring, and the use of surgical devices such as the LigaSure ${ }^{\mathrm{TM}}$ vessel sealer and divider (Impact ${ }^{\mathrm{TM}}$ and Small Jaw ${ }^{\mathrm{TM}}$ ), which facilitates the dissection, reduces the blood loss, and shortens the surgical time. ${ }^{4}$

Pelvic exenteration is an ultra-radical procedure characterized by en bloc resection of the pelvic organs and can be described on the three axes of the pelvis: antero-posterior, transversal and vertical. ${ }^{5-8}$ The antero-posterior axis includes an anterior pelvic exenteration of the upper rectum; the reproductive organs and the bladder are also removed, but the lower rectum may be spared, or a perineal excision may be performed. The posterior pelvic exenteration includes the removal of the rectum and reproductive system; the bladder may be saved, but removal of the sacrum or coccyx may be performed. Total pelvic exenteration involves the excision of the rectum, distal colon, genitourinary viscera-draining lymph nodes, pelvic peritoneum, and internal genital organs, and sacrectomy may be performed. The vertical method includes a supralevator exenteration (abdominal exenteration), infralevator exenteration (abdominoperineal exenteration or abdominosacral exenteration), exenteration with vulvectomy, or extended exenteration that adds the removal of an abdominal viscus.

The transverse approach includes exenteration outside the hypogastric vessels, which is the standard exenteration procedure; exenteration inside the pelvic plexus with the preservation of the innervation of the internal sphincter; exenteration extended to the lateral pelvic walls, with or without the removal of the sacral plexus; exenteration at the level of the hypogastric vessel, with or without lymph node dissection or the preservation of sphincter function.

The indications for this procedure include centrally recurrent cervical malignancies in patients who had undergone definitive radiotherapy, recurrent pelvic tumors if there is a chance to cure, and for control of local advanced cancers as a palliative procedure. ${ }^{9-12}$

Absolute contraindications for pelvic exenteration include the presence of remote metastases, tumor involvement of the common and external iliac arteries, para-aortic lymph node metastases, involvement of the sacrum or tumor extension within the sciatic foramen, as well as tumor extension to the pelvic sidewall. ${ }^{9-13}$ The relative contraindications of the procedure are ureteral barriers, associated disease that makes the patient a poor candidate for the op- eration, or patients that are unable to care for stomas or present senility. ${ }^{9-13}$

Supralevator exenterations can be performed if the tumor does not involve the lower, posterior third of the vagina, and if the distance between the tumor and levator muscles is $2 \mathrm{~cm}$ or greater. ${ }^{14}$

\section{CASE REPORT}

We report the case of a 54-year-old woman who presented in our department for pain in the lower abdominal floor, weight loss, nausea, and vomiting. From the personal medical history we found out that she had been diagnosed with cervical cancer stage IIB, for which she had been operated in 2015 with total hysterectomy with bilateral adnexectomy, followed by radio- and chemotherapy. She also suffered from a rectovaginal fistula diagnosed in March 2017, left ureteral lithiasis, left ureteral obstruction, and anemia.

Digital rectal and vaginal examination revealed a tumoral mass that was extending to the osseous walls on both sides. Sphincter function appreciated by the Wexner score was 8-9.

Computed tomography scan showed a recurrent cervical cancer invading the bladder and the rectum and left ureteral obstruction.

The cystoscopy revealed an extended fistula between the bladder and vagina, which included both ureteral orifices.

Due to left ureteral lithiasis and left ureteral obstruction, the patient was admitted to the Clinic of Urology of Tîrgu Mureș, where she underwent percutaneous nephrolithotomy followed by a left nephrostomy. After surgery, she presented a favorable evolution and was discharged. A month later the patient was admitted, under emergency conditions, to the Clinic of Urology with lumbar pain and dislocation of the nephrostoma. During hospitalization, the patient presented intense abdominal pain located in the lower abdominal quadrant, nausea, and vomiting, and after the repositioning of the left nephrostoma, she was transferred to the Surgical Clinic I of the County Emergency Clinical Hospital of Tîrgu Mureș.

After a preoperative preparation, a supralevator total pelvic exenteration was performed (Figure 1) with interaortocaval and pelvic lymphadenectomy, pelvic ureter resection on the right side with cutaneous ureterostomy, and total resection of the left ureter with remnant nephrostoma. The tumoral mass was removed "en bloc", including the rectum, bladder, and vagina, with a double tumoral fistula (recto-vaginal-bladder).

The reconstruction procedure included right ureterostomy, remnant left nephrostomy, vulvoplasty after total 


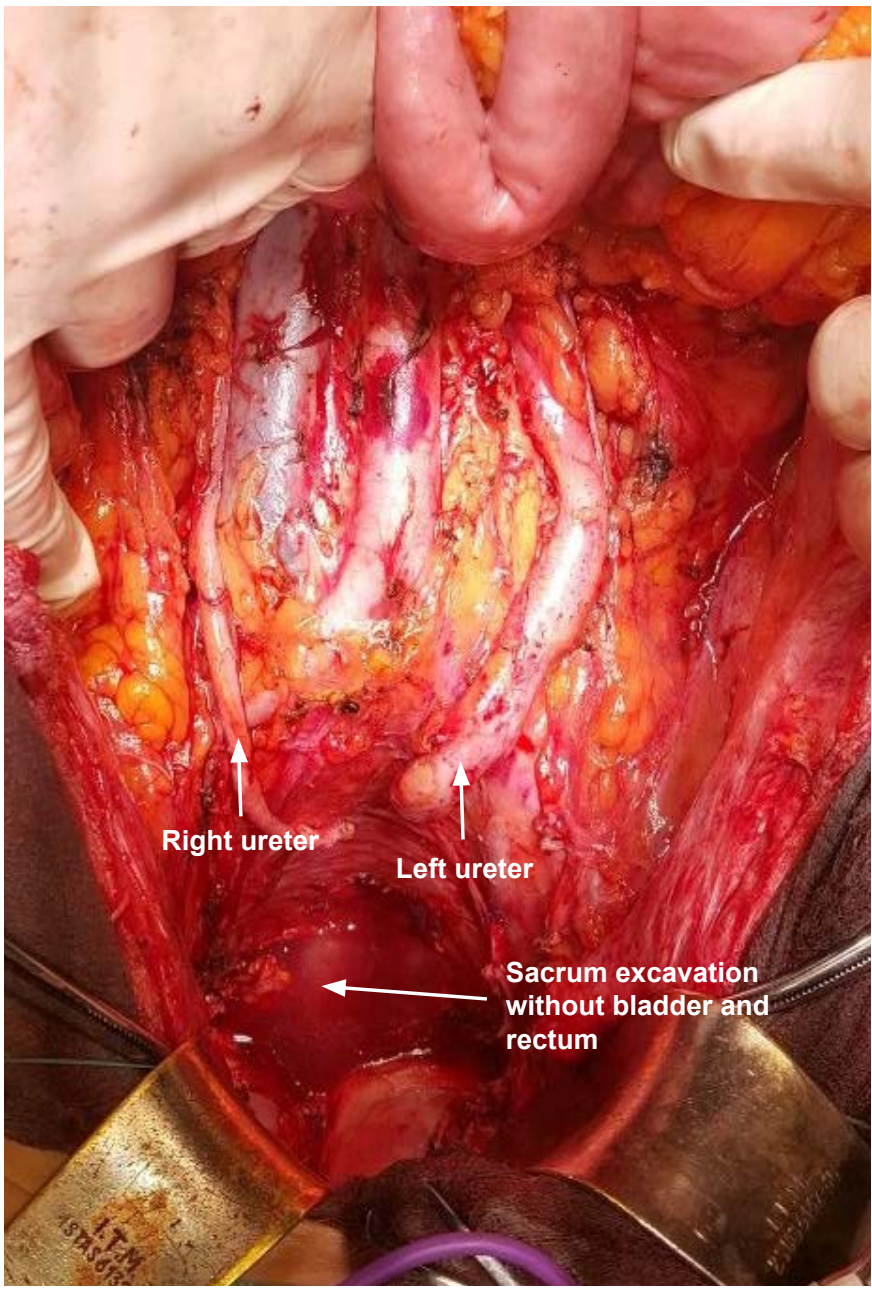

FIGURE 1. Supralevator total pelvic exenteration

colpectomy, and colocutaneous per-anal anastomosis using the Parks procedure (Figure 2).

The postoperative evolution was favorable, without surgical complications, with acceptable fecal and flatus continence (Wexner score 8), and good urinary function. The oncological treatment and outpatient follow-up was favorable, as the PET-CT results showed negative residual or metastatic neoplasia, and the biomarkers for cancer were also negative at 3 months following surgery. The patient agreed to the publication of her data and the institution where the patient had been admitted, approved the publication of the case.

\section{DISCUSSION}

Exenterations are complex surgeries that require a multidisciplinary team with participants from gynecology, urology, colorectal surgery, oncology, plastic surgery, and anesthesia. Our surgery team was formed by gynecologists, colorectal surgeons, and urology surgeons.

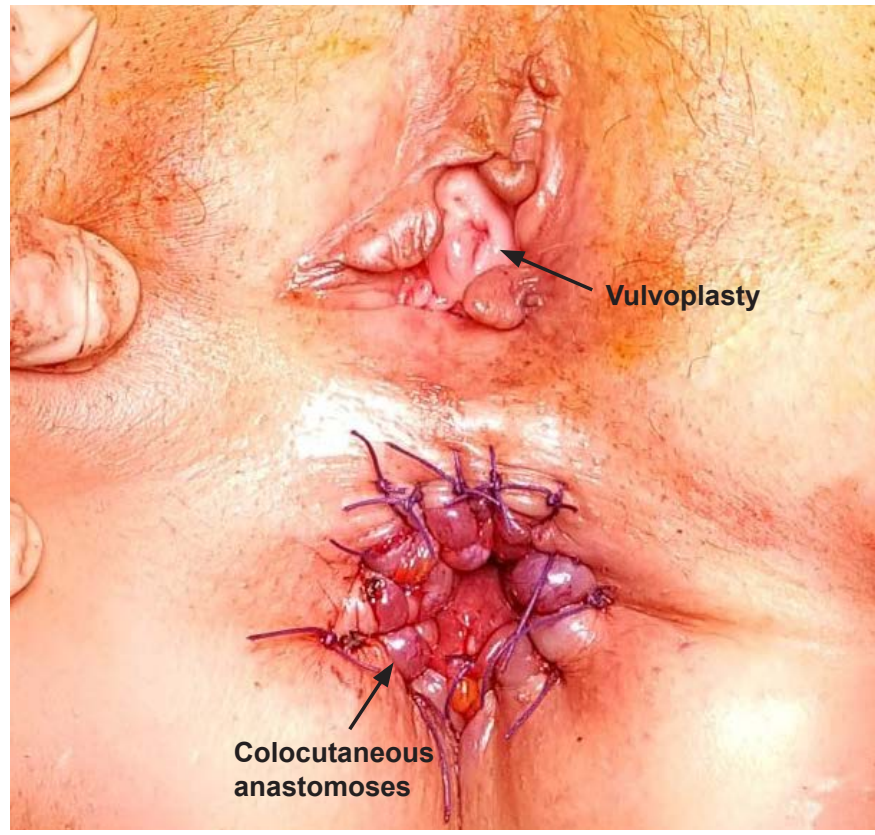

FIGURE 2. Colocutaneous anastomosis

During the surgery, vessel sealer and divider instruments such as the LigaSure ${ }^{\mathrm{TM}}$ (Impact ${ }^{\mathrm{TM}}$ and Small Jaw ${ }^{\mathrm{TM}}$ ) were used, which facilitated the dissection, reduced the blood loss, and shortened the surgery time.

The presence of digestive and urinary stomas is the main factor that affects the quality of life of patients with pelvic exenterations. Supralevator exenterations can be concluded with low colorectal anastomosis in most of patients. Almost all authors recommend a protective colostomy for irradiated patients and when pelvic infections occur. By restoring the anal transit by a Parks colocutaneous anastomosis, both the protective stoma and the risk for anastomotic fistulas were avoided. In fact, a "perineal sphincter stoma” was achieved, with a good contractile function.

\section{CONCLUSION}

Supralevator exenteration provides hope for cure in patients with pelvic cancers that recur after radiation therapy. The restoration of the anal transit using a Parks colocutaneous anastomosis increases the quality of life in patients with pelvic exenteration by avoiding the colostomy. The selection of patients for these sphincter-saving procedures is highly important in providing the best results in such cases.

\section{CONFLICT OF INTEREST}

Nothing to declare. 


\section{REFERENCES}

1. Brunschwig A. Complete excision of pelvic viscera for advanced carcinoma; a one-stage abdominoperineal operation with end colostomy and bilateral ureteral implantation into the colon above the colostomy. Cancer. 1948;1:177-183.

2. Benn T, Brooks RA, Zhang Q, et al. Pelvic exenteration in gynecologic oncology: a single institution study over 20 years. Gynecol Oncol. 2011;122:14-18

3. Heriot $A G$, Byrne $C M$, Lee $P$, et al. Extended radical resection: the choice for locally recurrent rectal cancer. Dis Colon Rectum. 2008;51:284-291.

4. Caceres A, Mourton SM, Bochner $\mathrm{BH}$, et al. Extended pelvic resections for recurrent uterine and cervical cancer: out-of-the-box surgery. International Journal of Gynecological Cancer. 2008;18:1139-1144.

5. Micha JP, Goldstein BH, Rettenmaier MA, Brown JV. Cecal pelvic transposition following total pelvic exenteration. Gynecol Oncol. 2004:94:589-592.

6. Hawighorst-Knapstein S, Fusshoeller C, et al. The impact of treatment for genital cancer on quality of life and body image-results of a prospective longitudinal 10 years study. Gynecol Oncol. 2004;94:398-403.

7. Magrina JF. Types of pelvic exenteration: a reappraisal. Gynecol Oncol. 1990;37:363-366
8. Magrina JF, Stanhope CR, Weaver AL. Pelvic exenterations: supralevator, infralevator, and with vulvectomy. Gynecol Oncol. 1997;64:130-135.

9. Gannon CJ, Zager JS, Chang GJ, et al. Pelvic exenteration affords safe and durable treatment for locally advanced rectal carcinoma. Ann Surg Oncol. 2007;14:1870-1877.

10. Lopes A, Poletto AH, Carvalho AL, Ribeiro EA, Granja NM, Rossi BM. Pelvic exenteration and sphincter preservation in the treatment of soft tissue sarcomas. Eur J Surg Oncol. 2004;30:972-975.

11. Kecmanovic DM, Pavlov MJ, Kovacevic PA, Sepetkovski AV, Ceranic MS, Stamenkovic AB. Management of advanced pelvic cancer by exenteration. Eur J Surg Oncol. 2003;29:743-746.

12. Pathiraja P, Sandhu H, Instone M, Haldar K, Kehoe S. Should pelvic exenteration for symptomatic relief in gynaecology malignancies be offered? Arch Gynecol Obstet. 2014;289:657-662.

13. Höckel M. Laterally extended endopelvic resection. Novel surgical treatment of locally recurrent cervical carcinoma involving the pelvic side wall. Gynecol Oncol. 2003;91:369-377.

14. Sagebiel TL, Viswanathan C, Patnana M, Devine CE, Frumovitz M, Bhosale PR. Overview of the Role of Imaging in Pelvic Exenteration. Radio Graphics. 2015;35:1286-1294. 